\title{
ROBUST FAULT DETECTION OF SINGULAR LPV SYSTEMS WITH MULTIPLE TIME-VARYING DELAYS
}

\author{
Amir Hossein HASSANABADI ${ }^{a}$, MASOUd SHAFIEE $^{a, b, *}$, VICENÇ PUIG $^{c}$ \\ ${ }^{a}$ Department of Electrical Engineering \\ Amirkabir University of Technology (Tehran Polytechnic), 424, Hafez Av., Tehran, Iran \\ e-mail: \{a.hassanabadi, mshafiee @aut.ac.ir \\ ${ }^{b}$ System, Information and Control Research Center \\ Amirkabir University of Technology (Tehran Polytechnic), 424, Hafez Ave., Tehran, Iran \\ ${ }^{c}$ Advanced Control Systems Group (SAC) \\ Universitat Politècnica de Catalunya (UPC), Pau Gargallo, 5, 08028 Barcelona, Spain \\ e-mail: vicenc.puig@upc.edu
}

\begin{abstract}
In this paper, the robust fault detection problem for LPV singular delayed systems in the presence of disturbances and actuator faults is considered. For both disturbance decoupling and actuator fault detection, an unknown input observer (UIO) is proposed. The aim is to compute a residual signal which has minimum sensitivity to disturbances while having maximum sensitivity to faults. Robustness to unknown inputs is formulated in the sense of the $\mathcal{H}_{\infty}$-norm by means of the bounded real lemma (BRL) for LPV delayed systems. In order to formulate fault sensitivity conditions, a reference model which characterizes the ideal residual behavior in a faulty situation is considered. The residual error with respect to this reference model is computed. Then, the maximization of the residual fault effect is converted to minimization of its effect on the residual error and is addressed by using the BRL. The compromise between the unknown input effect and the fault effect on the residual is translated into a multi-objective optimization problem with some LMI constraints. In order to show the efficiency and applicability of the proposed method, a part of the Barcelona sewer system is considered.
\end{abstract}

Keywords: singular delayed LPV systems, fault detection, unknown input observer (UIO), robustness, fault sensitivity.

\section{Introduction}

Modern systems have become more complex and the need for higher performance and reliability has made fault diagnosis a crucial element. The basis for model-based fault diagnosis methods is to consider a mathematical model of the system, comparing the outputs of this model with real outputs (measurements), and finally trying to discover any abnormal situation in the system through finding an inconsistency between these two signals (Chen and Patton, 1999).

Normally, a signal called residual is generated based on the difference between the real and model outputs, which in the ideal case is zero but becomes nonzero in the faulty case. Unknown inputs and modeling errors cause this signal to be nonzero in healthy conditions, so the

* Corresponding author residual should be compared with a threshold instead of zero. Due to the effect of unknown input and modeling errors on the residual generator system, the robustness of fault diagnosis methods is an important issue to be considered.

Robustness can be taken into account at the residual generation stage using several approaches, such as unknown input observers (UIOs) in the work of Guan and Saif (1991), or using adaptive thresholds in the residual evaluation stage (Montes de Oca et al., 2012a). Observer-based methods for fault diagnosis and unknown input robustness in these methods have been well established for LTI systems (see the monograph by Ding (2008) and the references therein). Some of the approaches are based on the UIO (Guan and Saif, 1991) or on robust observers in the sense of the $\mathcal{H}_{\infty}$-norm (Hou and Patton, 1996). Hamdi et al. (2012b) and Youssef 
et al. (2014) designed proportional integral type UIOs which make it possible to estimate unknown inputs in the system. In addition to robustness, the residual should present good sensitivity to faults. Various methods such as model matching the residual with a reference model (Frisk and Nielsen, 2006; Mazars et al., 2008; Ichalal et al., 2009; Habib et al., 2010; Ahmadizadeh et al., 2014) and using the $\mathcal{H}_{-}$index condition to directly formulate fault sensitivity (Wang et al., 2007; Aouaouda et al., 2015; Zhai et al., 2014) were proposed for ensuring fault sensitivity in observer-based methods.

Time delay occurs in many systems either because of delayed actuation or measurement or because of delay in internal states of the system. Delay in system dynamics usually degrades the stability and performance of the system. Control and diagnosis of time delay systems require more involved conditions than the systems without delay and are still active research areas. The current research in time delay systems aims at developing conditions for various control problems such as stabilization or observer design that are less conservative than the existing ones. Saadni et al. (2005) and Liu et al. (2016) provided delay dependent conditions for stabilization, while Karimi and Chadli (2012) introduced less conservative conditions for observer design. In the case of state delay systems, UIO design was considered by Fattouh et al. (1999) and Fu et al. (2004), while an $\mathcal{H}_{\infty}$ fault detection filter for robust fault detection was studied by Bai et al. (2006). In the case of input delay systems, a UIO which is used for fault detection and isolation was proposed by Koenig et al. (2005). A geometric approach to fault detection in time-delay systems was put forward by Meskin and Khorasani (2009). In the work of Ahmadizadeh et al. (2014), a UIO was designed for fault detection in uncertain time delay systems.

Descriptor systems are a more general class of systems compared with standard state space ones since they allow modeling systems, including static and dynamic equations, simultaneously (Dai, 1989; Duan, 2010). They are also called differential-algebraic equations (DAEs) for their modeling power and singular systems for the singular nature of the equations that arise from the modeling. Singular systems have been successfully used in modeling and analysis of many robotic systems (Samiei and Shafiee, 2010; Sadati et al., 2013) and also in other systems such as electrical (Hamdi et al., 2012a; Zhai et al., 2014) and economic ones (Koumboulis et al., 2011). A lot of effort has been devoted to stability analysis, observer design and control of these systems (see the monograph by Duan (2010) and the references therein). However, fault diagnosis in descriptor systems is still in its developmental stage. An eigenstructure approach was used to design a UIO by Duan et al. (2002). A UIO was proposed by Yeu et al. (2005) for fault detection and isolation, and a UIO was designed for fault estimation by Koenig (2005). A singular UIO and a fault detection filter for diagnosis purposes in these systems were designed respectively by Share Pasand et al. (2010) and Yao et al. (2011).

In the case of linear singular systems which have delayed dynamics, scarce research has been done for fault detection and isolation. Two robust fault detection filter schemes for these systems were introduced by Chen $e t a l$. (2011) and Zhai et al. (2014). For nonlinear singular delayed systems, to the best of the authors' knowledge, no work has been carried out addressing fault diagnosis. Simultaneous presence of nonlinearity and a delayed term added by the singular nature of these systems increases the demand for a particular treatment.

One of the usual methodologies to treat nonlinear systems is to consider special classes, such as those satisfying the Lipschitz condition. However, this condition limits the range of applicability of the obtained results. Modeling nonlinear systems with some linear ones at different operating points is a common idea that is used to treat nonlinear systems. Based on this idea, Shamma (1988) proposed linear parameter varying (LPV) systems with a linear structure but with the state space matrices depending on some exogenous parameters.

In the work of Bokor and Balas (2004), a geometric approach was used to design the fault detection filter for LPV systems. Henry et al. (2009) used the $\mathcal{H}_{\infty} / \mathcal{H}_{-}$ approach to address simultaneous fault sensitivity and robustness of a residual in LPV systems. A fault tolerant controller in LPV systems based on a UIO fault estimator was designed by Montes de Oca et al. (2012b). UIO design based on perfect and robust unknown input decoupling for singular LPV systems was considered respectively by Hamdi et al. (2012b) and Habib et al. (2010). Fault diagnosis in these systems in the case of unmeasurable scheduling parameters was discussed by Lopez-Estrada et al. (2014). For the case of singular LPV systems with delay, the problem of fault detection has not been considered yet.

The main contribution in this paper is to address the problem of robust fault detection of singular delayed LPV systems including multiple time varying state delays and disturbances. The system under consideration also includes actuator faults. After converting the system to polytopic representation, a UIO is proposed. The presence of unknown inputs in the system involves simultaneous robustness and fault sensitivity treatment in the fault detection process. Unknown input attenuation is considered with a bounded real lemma (BRL) for delayed LPV systems. The fault sensitivity issue is considered by means of a reference model which models the ideal residual behavior with respect to a fault (Mazars et al., 2008). The residual error compared with the output of this reference model is computed and the 
fault sensitivity objective is reformulated into fault effect attenuation on the residual error. Then, this objective can be formulated in a standard framework using a BRL for the residual error augmented system. In particular, the trade-off between robustness and fault sensitivity is addressed in a multi-objective optimization problem with LMI constraints. To illustrate the performance of the proposed approach, a part of the Barcelona sewer network is considered as a case study.

Sewer networks are complex large-scale systems which combine sanitary and rain water flows within the same network in most cities around the world (Puig et al., 2009; Ocampo Martínez et al., 2013). They behave as open-flow channel systems. As discussed by Bolea et al. (2014), these systems present dynamic behavior that can be represented by means of a delayed LPV model. When a network of open flow channels is considered, a singular delayed LPV model should be used to take into account the mass balances in the nodes. Moreover, during rain storms, wastewater flows can easily overload the sewer network capacity, thereby causing operators to dump the excess of water into the nearest receiver environment (rivers, streams or sea). Highly sophisticated supervisory-control systems are used to ensure that high performance can be achieved and maintained under adverse conditions. The need to operate in adverse meteorological conditions involves, with a high probability, sensor and actuator malfunctions (faults). This problem calls for the use of an on-line fault detection and isolation (FDI) system able to detect such faults and correct them (if possible) by activating fault tolerance mechanisms (Puig and Blesa, 2013).

The remainder of the paper is organized as follows: In Section 2, the problem is formulated. In Section 3 , the structure of a UIO for the systems under consideration is proposed. In Section 4 simultaneous robustness and fault sensitivity is formulated with the aid of a reference model. The trade-off is considered in a multi-objective optimization problem with LMI constraints. In Section 5. a part of the Barcelona sewer system is discussed as a real application case study, where the method proposed in this paper is applied for actuator fault detection and disturbance decoupling. Section 6 draws the main conclusions.

Notation. Throughout this paper, the following notation is used: $\mathbb{R}$ is the set of real numbers; $I_{n}$ is the $n$-dimensional identity matrix; for a matrix $X, X^{T}$ signifies its transpose; $X^{-1}$ is the inverse and $X^{+}$is the pseudoinverse of $X$; for a symmetric matrix, $*$ is used to show the elements induced from symmetry; for a symmetric matrix $X, X>$ $0(X<0)$ shows that it is positive (negative) definite. For a square integrable function $x(t)$, its $\mathcal{L}_{2}$-norm is defined as $\|x(t)\|_{2}=\sqrt{\int_{0}^{\infty} x(t)^{T} x(t) \mathrm{d} t}$.

\section{Problem formulation}

This paper considers singular delayed LPV systems including disturbances and actuator faults that can be formulated in state-space form as follows:

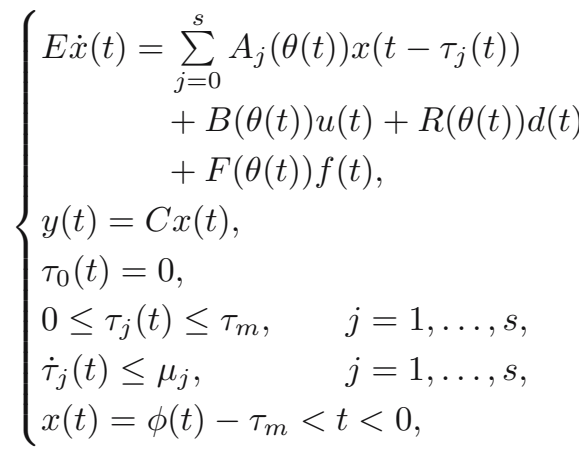

where $x(t) \in \mathbb{R}^{n}, u(t) \in \mathbb{R}^{k_{u}}, y(t) \in \mathbb{R}^{m}, d(t) \in$ $\mathbb{R}^{k_{d}}$ and $f(t) \in \mathbb{R}^{k_{f}}$ are the state vector, input vector, output vector, exogenous disturbances and actuator faults, respectively. In (1), $E \in \mathbb{R}^{n \times n}$ is a constant square matrix that may have rank deficiency $(\operatorname{rank}(E)=r \leq$ $n)$. $A_{j}(\theta(t))$ for $j=0, \ldots, s, B(\theta(t)), R(\theta(t))$ and $F(\theta(t))$ are matrices with the appropriate dimensions which depend affinely on the time varying parameter $\theta(t) \in \mathbb{R}^{l}$ that is real time measurable. $C$ is a constant matrix with appropriate dimensions. Here $\tau_{j}(t)$ for $j=1, \ldots, s$ are time varying delays and $\tau_{0}(t)=0$. Furthermore, $\tau_{m}$ is the maximum bound on all delay values, and $\mu_{j}$ for $j=1, \ldots, s$ are the maximum bounds on delay derivative values. $\phi(t)$ is a continuous vector-valued initial function. The time varying parameter vector $\theta(t)$ is assumed to be bounded in a hyperbox (Hamdi et al., 2012b), i.e.,

$$
\theta_{k}^{m} \leq \theta_{k}(t) \leq \theta_{k}^{M}, \quad k=1, \ldots, l .
$$

Definition 1. (Dai, 1989; Duan, 2010) The matrix pencil $(E, A)$ is regular if $\operatorname{det}(s E-A)$ is not identically zero.

Definition 2. (Dai, 1989; Duan, 2010) The matrix pencil $(E, A)$ is impulse-free if $\operatorname{deg}(\operatorname{det}(s E-A))=\operatorname{rank}(E)$.

Definition 3. (Li and Zhang, 2012) System (1) is regular and impulse-free if the matrix pencil $\left(E, A_{0}(\theta(t))\right.$ is regular and impulse-free for the whole domain of $\theta(t)$ defined in (2).

Definition 4. (Li and Zhang, 2012) The system (1) is admissible if it is regular, impulse free and stable.

Remark 1. ( $\mathrm{Li}$ and Zhang, 2012) Regularity and impulse-freeness of the system (1) guarantee a unique continuous solution without impulsive behavior in the case of a compatible initial function $\phi(t)$ for the system (1). 
Assumption 1. The singular delayed LPV system considered in this paper is admissible according to the definitions provided.

Thus, admissibility, stabilization and control of this system are not addressed in this paper. The reader interested in these issues is referred to the works of $\mathrm{Li}$ and Zhang (2012) as well as Zhang and Zhu (2012).

The system (1) can be transformed to a polytopic representation. In the polytopic description, $h=2^{l}$ subsystems are considered in $h$ vertices of the hyperbox in the $\theta(t)$ space and the whole system is defined as a convex combination of these subsystems. According to the theory of convex sets, each point in the $\theta(t)$ space can be represented by a set of subsystem weights defined on $h$ vertices of the hyperbox in this space. These weights are considered here by $\rho(\theta(t)) \in \mathbb{R}^{h}$ and satisfy

$$
\begin{array}{ll}
0 \leq \rho_{i}(\theta(t)) \leq 1, & i=1, \ldots, h, \\
\sum_{i=1}^{h} \rho_{i}(\theta(t)) & =1, \quad i=1, \ldots, h .
\end{array}
$$

Thus, the polytopic representation of (1) is

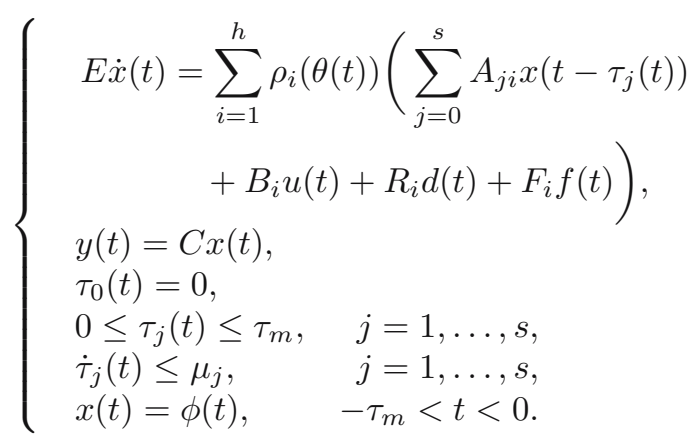

In (5), $A_{j i}, B_{i}, R_{i}$ and $F_{i}$ are matrices related to the $i$-th subsystem $(i=1, \ldots, h)$ located at the $i$-th vertex of the hyperbox.

\section{UIO structure}

Considering the polytopic system (5), the following observer is proposed:

$$
\left\{\begin{aligned}
\dot{z}(t)= & \sum_{i=1}^{h} \rho_{i}(\theta(t))\left(\sum _ { j = 0 } ^ { s } \left(N_{j i} z\left(t-\tau_{j}(t)\right)\right.\right. \\
& \left.\left.+L_{j i} y\left(t-\tau_{j}(t)\right)\right)+G_{i} u(t)\right) \\
\hat{x}(t)= & z(t)+H_{2} y(t) \\
\hat{y}(t)= & C \hat{x}(t) \\
r(t)= & T(y(t)-\hat{y}(t)) \\
z(t)= & 0, \quad-\tau_{m}<t<0
\end{aligned}\right.
$$

where $\hat{x}(t) \in \mathbb{R}^{n}, \hat{y}(t) \in \mathbb{R}^{m}$ and $z(t) \in \mathbb{R}^{n}$ are the state estimate, output estimate and observer state, respectively.
$N_{j i}, L_{j i}, G_{i}, T$ and $H_{2}$ are observer matrices with appropriate dimensions that will be determined with the proposed design methodology later on. Here $r(t) \in \mathbb{R}^{k_{r}}$ is the residual signal which is used for detecting the faults in the system. The state estimation error is

$$
e(t)=x(t)-\hat{x}(t) .
$$

The error dynamics will be designed so that the error converges to zero under arbitrary initial conditions and any input $u(t)$. According to (6), the error $e(t)$ in (7) is

$$
\begin{aligned}
e(t) & =x(t)-z(t)-H_{2} C x(t) \\
& =\left(I_{n}-H_{2} C\right) x(t)-z(t) .
\end{aligned}
$$

If $H_{1} \in \mathbb{R}^{n \times n}$ can be found such that the condition

$$
H_{1} E=I_{n}-H_{2} C,
$$

is satisfied (Hamdi et al., 2012b) then

$$
e(t)=H_{1} E x(t)-z(t) .
$$

Thus, the error dynamics are described by means of

$$
\dot{e}(t)=H_{1} E \dot{x}(t)-\dot{z}(t) .
$$

Substituting (5) and (6) in (11) results in

$$
\begin{aligned}
\dot{e}(t)= & \sum_{i=1}^{h} \rho_{i}(\theta(t))\left(\sum _ { j = 0 } ^ { s } \left(H_{1} A_{j i} x\left(t-\tau_{j}(t)\right)\right.\right. \\
& \left.-N_{j i} z\left(t-\tau_{j}(t)\right)-L_{j i} C x\left(t-\tau_{j}(t)\right)\right) \\
& +H_{1} B_{i} u(t)+H_{1} R_{i} d(t) \\
& \left.+H_{1} F_{i} f(t)-G_{i} u(t)\right)
\end{aligned}
$$

and, according to 10 , observing that

$$
\begin{aligned}
& -N_{j i} z\left(t-\tau_{j}(t)\right) \\
& \quad=N_{j i} e\left(t-\tau_{j}(t)\right)-N_{j i} H_{1} E x\left(t-\tau_{j}(t)\right),
\end{aligned}
$$

(12) can be written as follows:

$$
\begin{aligned}
& \dot{e}(t) \\
& =\sum_{i=1}^{h} \rho_{i}(\theta(t))\left(\sum _ { j = 0 } ^ { s } \left(N_{j i} e\left(t-\tau_{j}(t)\right)\right.\right. \\
& \left.\quad+\left(H_{1} A_{j i}-L_{j i} C-N_{j i} H_{1} E\right) x\left(t-\tau_{j}(t)\right)\right) \\
& \left.\quad+\left(H_{1} B_{i}-G_{i}\right) u(t)+H_{1} R_{i} d(t)+H_{1} F_{i} f(t)\right) .
\end{aligned}
$$

If the following conditions are satisfied for $i=$ $1, \ldots, h$ and $j=0, \ldots, s$ :

$$
H_{1} E+H_{2} C=I_{n},
$$




$$
\begin{gathered}
H_{1} A_{j i}-N_{j i} H_{1} E=L_{j i} C, \\
G_{i}=H_{1} B_{i},
\end{gathered}
$$

the residual dynamic system can be transformed into

$$
\left\{\begin{aligned}
\dot{e}(t)= & \sum_{i=1}^{h} \rho_{i}(\theta(t))\left(\sum_{j=0}^{s} N_{j i} e\left(t-\tau_{j}(t)\right)\right. \\
& \left.+H_{1} F_{i} f(t)+H_{1} R_{i} d(t)\right) \\
r(t)= & T C e(t) .
\end{aligned}\right.
$$

Now, a lemma is introduced for robust stability of a delayed LPV system that will be used for robust fault detection of the system (5).

Lemma 1. Consider the following delayed LPV system:

$$
\left\{\begin{aligned}
\dot{e}(t)= & \sum_{i=1}^{h} \rho_{i}(\theta(t)) \\
& \times\left(\sum_{j=0}^{s} \bar{A}_{j i} e\left(t-\tau_{j}(t)\right)+\bar{B}_{i} w(t)\right), \\
z(t)= & \bar{C} e(t)+\bar{D} w(t),
\end{aligned}\right.
$$

in which $w(t)$ is a $\mathcal{L}_{2}$-norm bounded exogenous input and $z(t)$ is the measured output, and all matrices have compatible dimensions. For a given $\gamma>0$, if there exist $P>0$ and $Q_{j}>0$ for $j=1, \ldots, s$ such that the following matrix inequality holds for $i=1, \ldots, h$ :

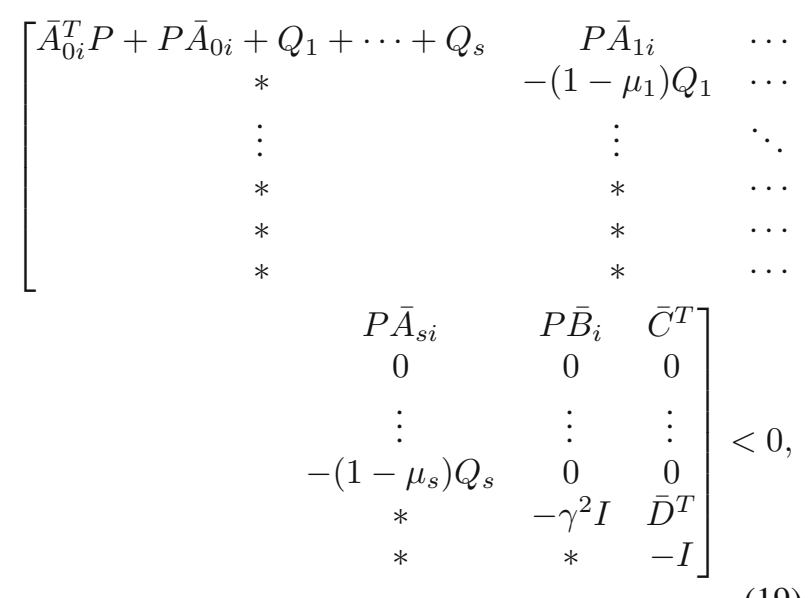

then the system (18) is asymptotically stable for $w(t)=0$ and $\|z(t)\|_{2}<\gamma\|w(t)\|_{2}$ for zero initial conditions.

Proof. The following Lyapunov-Krasovskii functional is considered:

$$
\begin{aligned}
V\left(t, e_{t}\right)= & e^{T}(t) P e(t) \\
& +\sum_{j=1}^{s} \int_{t-\tau_{j}(t)}^{t} e^{T}(\lambda) Q_{j} e(\lambda) \mathrm{d} \lambda
\end{aligned}
$$

in which $P$ and $Q_{j}(j=1, \ldots, s)$ are symmetric positive definite matrices and $e_{t}:=e(t+\omega)$, where $\omega \in\left[-\tau_{m}, 0\right]$. Consider the index

$$
J_{2}=\int_{0}^{\infty}\left(z(t)^{T} z(t)-\gamma^{2} w(t)^{T} w(t)\right) \mathrm{d} t
$$

Then the disturbance attenuation condition $\|z(t)\|_{2}<$ $\gamma\|w(t)\|_{2}$ in this lemma is equivalent to $J_{2}<0$. Thus, for proving the lemma, it is just necessary to show that $J_{2}<0$ for nonzero $w(t)$ with zero initial conditions and $\dot{V}\left(t, e_{t}\right)<0$ for zero $w(t) . J_{2}$ can be written as follows:

$$
\begin{aligned}
J_{2}= & \int_{0}^{\infty}\left(z(t)^{T} z(t)-\gamma^{2} w(t)^{T} w(t)+\dot{V}\left(t, e_{t}\right)\right) \mathrm{d} t \\
& +\left.V\left(t, e_{t}\right)\right|_{t=0}-\left.V\left(t, e_{t}\right)\right|_{t=\infty} .
\end{aligned}
$$

Under zero initial conditions, $\left.V\left(t, e_{t}\right)\right|_{t=0}=0$ by the definition of the Lyapunov-Krasovskii functional and $\left.V\left(t, e_{t}\right)\right|_{t=\infty} \geq 0$ owing to the positive definiteness of this functional, and it follows that

$$
\begin{aligned}
J_{2} \leq & \int_{0}^{\infty}\left(z(t)^{T} z(t)-\gamma^{2} w(t)^{T} w(t)+\dot{V}\left(t, e_{t}\right)\right) \mathrm{d} t \\
= & \int_{0}^{\infty}\left(e^{T}(t) \bar{C}^{T} \bar{C} e(t)+e^{T}(t) \bar{C}^{T} \bar{D} w(t)\right. \\
& +w^{T}(t) \bar{D}^{T} \bar{C} e(t)+w^{T}(t) \bar{D}^{T} \bar{D} w(t) \\
& \left.-\gamma^{2} w(t)^{T} w(t)\right) \mathrm{d} t \\
& +\int_{0}^{\infty} \sum_{i=1}^{h} \rho_{i}(\theta(t))\left(e^{T}(t) P \bar{A}_{0 i} e(t)\right. \\
& +e^{T}(t) \bar{A}_{0 i}^{T} P e(t)+\sum_{j=1}^{s}\left(e^{T}(t) P \bar{A}_{j i} e\left(t-\tau_{j}(t)\right)\right. \\
& \left.+e^{T}\left(t-\tau_{j}(t)\right) \bar{A}_{j i}^{T} P e(t)\right)+e^{T}(t) P \bar{B}_{i} w(t) \\
& +w^{T}(t) \bar{B}_{i}^{T} P e(t)+\sum_{j=1}^{s}\left(e^{T}(t) Q_{j} e(t)\right. \\
& \left.\left.-\left(1-\dot{\tau}_{j}(t)\right) e^{T}\left(t-\tau_{j}(t)\right) Q_{j} e\left(t-\tau_{j}(t)\right)\right)\right) \mathrm{d} t .
\end{aligned}
$$

Since

$$
\sum_{i=1}^{h} \rho_{i}(\theta(t))=1
$$

and $\dot{\tau}_{j} \leq \mu_{j}$ for $j=1, \ldots, s$, the previous expression can 
be bounded as follows:

$$
\begin{gathered}
J_{2} \leq \int_{0}^{\infty} \sum_{i=1}^{h} \rho_{i}(\theta(t))\left[\begin{array}{c}
e(t) \\
e\left(t-\tau_{1}(t)\right) \\
\vdots \\
e\left(t-\tau_{s}(t)\right) \\
w(t)
\end{array}\right]^{T} \Theta \\
\times\left[\begin{array}{c}
e(t) \\
e\left(t-\tau_{1}(t)\right) \\
\vdots \\
e\left(t-\tau_{s}(t)\right) \\
w(t)
\end{array}\right] \mathrm{d} t,
\end{gathered}
$$

where

$$
\begin{gathered}
\Theta=\left[\begin{array}{ccc}
\Xi & P \bar{A}_{1 i} & \cdots \\
* & -\left(1-\mu_{1}\right) Q_{1} & \cdots \\
\vdots & \vdots & \ddots \\
* & * & \cdots \\
* & * & \cdots \\
P \bar{A}_{s i} & P \bar{B}_{i}+\bar{C}^{T} \bar{D} \\
0 & 0 \\
\vdots & \vdots \\
-\left(1-\mu_{s}\right) Q_{s} & 0 \\
* & \bar{D}^{T} \bar{D}-\gamma^{2} I
\end{array}\right], \\
\Xi=\bar{A}_{0 i}^{T} P+P \bar{A}_{0 i}+\bar{C}^{T} \bar{C}+Q_{1}+\cdots+Q_{s} .
\end{gathered}
$$

Then, by using the Schur complement lemma, (19) can be obtained which is a sufficient condition for $J_{2}<0$ under zero initial conditions. The feasibility of the LMI:

$$
\left[\begin{array}{ccc}
\bar{A}_{0 i}^{T} P+P \bar{A}_{0 i}+Q_{1}+\cdots+Q_{s} & P \bar{A}_{1 i} & \cdots \\
* & -\left(1-\mu_{1}\right) Q_{1} & \cdots \\
\vdots & \vdots & \ddots \\
* & * & \cdots \\
& P \bar{A}_{s i} \\
0 \\
\vdots \\
\\
& -\left(1-\mu_{s}\right) Q_{s}
\end{array}\right]<0
$$

is directly deduced from (19), which insures $\dot{V}\left(t, e_{t}\right)<0$ for zero $w(t)$, so the system 18 is asymptotically stable under no actuation.

Remark 2. The BRL introduced in Lemma 1 for robust stability of the delayed LPV system (18) is dependent on delay derivatives but independent of delay values. There are some results in the literature which present delay dependent conditions (see the works of Saadni et al. (2005), Wu et al. (2010) and Liu et al. (2016) for delay dependent stability and stabilization results). However, the goal of Lemma 1 is to introduce a robust stability result for the system (18) which can be directly used in the remaining parts of the paper, for the design of a robust fault detection observer of a singular delayed LPV system in the LMI format. Other robust stability results in the literature cannot be directly used since the LMI formulation of the present method involves dealing with some nonlinear terms in matrix inequalities, which is beyond the scope of this paper.

\section{Robust fault detection system design}

Now, the design of the observer (6) is considered such that the conditions (14) - 16) are met and the error dynamics (17) will be robustly stable while having maximum sensitivity to the actuator fault based on Lemma 1.

Assumption 2. For the solvability of (14), it is assumed that

$$
\operatorname{rank}\left[\begin{array}{c}
E \\
C
\end{array}\right]=n
$$

Under Assumption 2, the solution of the matrix equation (14) is (Darouach and Boutayeb, 1995; Hamdi et al., 2012b)

$$
\left[\begin{array}{ll}
H_{1} & H_{2}
\end{array}\right]=\left[\begin{array}{l}
E \\
C
\end{array}\right]^{+},
$$

where

$$
\left[\begin{array}{l}
E \\
C
\end{array}\right]^{+} \in \mathbb{R}^{n \times(n+m)}
$$

is the pseudoinverse of $\left[\begin{array}{c}E \\ C\end{array}\right]$ calculated from

$$
\left[\begin{array}{l}
E \\
C
\end{array}\right]^{+}=\left(\left[\begin{array}{l}
E \\
C
\end{array}\right]^{T}\left[\begin{array}{l}
E \\
C
\end{array}\right]\right)^{-1}\left[\begin{array}{l}
E \\
C
\end{array}\right]^{T} .
$$

For extending the solution domain of (14), a term is added in the null space of this equation, so that

$$
\begin{aligned}
& {\left[\begin{array}{ll}
H_{1} & H_{2}
\end{array}\right]} \\
& \quad=\left[\begin{array}{l}
E \\
C
\end{array}\right]^{+}+K\left(I_{n+m}-\left[\begin{array}{l}
E \\
C
\end{array}\right]\left[\begin{array}{l}
E \\
C
\end{array}\right]^{+}\right),
\end{aligned}
$$

where $K \in \mathbb{R}^{n \times(n+m)}$ is a gain factor that can be freely chosen to satisfy other restrictions on the problem. Now (28) is rewritten as follows:

$$
\begin{aligned}
& H_{1}=H_{10}+K X_{1}, \\
& H_{2}=H_{20}+K X_{2},
\end{aligned}
$$

where $H_{10} \in \mathbb{R}^{n \times n}$ and $H_{20} \in \mathbb{R}^{n \times m}$ are constructed from the first $n$ columns and last $m$ columns of $\left[\begin{array}{l}E \\ C\end{array}\right]^{+}$ respectively. In a similar way, $X_{1} \in \mathbb{R}^{(n+m) \times n}$ and 
$X_{2} \in \mathbb{R}^{(n+m) \times m}$ are made from the first $n$ columns and last $m$ columns of

$$
X=I_{n+m}-\left[\begin{array}{l}
E \\
C
\end{array}\right]\left[\begin{array}{l}
E \\
C
\end{array}\right]^{+},
$$

respectively.

Until now, the condition (14) has been considered for observer existence. On the other hand, the condition (15) should be met such that the error dynamics will have the desired characteristic of being robust to disturbances and sensitive to faults. Now, some new variables are introduced for $j=0, \ldots, s$ and $i=1, \ldots, h$ :

$$
K_{j i}=L_{j i}-N_{j i} H_{2} .
$$

With these new variables, the condition (15) can be rewritten as

$$
N_{j i}=H_{1} A_{j i}-K_{j i} C .
$$

Substituting (29) in (32) results in

$$
N_{j i}=H_{10} A_{j i}+K X_{1} A_{j i}-K_{j i} C .
$$

With this methodology, whenever $K$ and $K_{j i}$ are found, $N_{j i}$ can be calculated from (33) and then, according to 31 ,

$$
L_{j i}=K_{j i}+N_{j i} H_{2} .
$$

Substituting (33) in 17) results in

$$
\left\{\begin{aligned}
\dot{e}(t)= & \sum_{i=1}^{h} \rho_{i}(\theta(t)) \\
& \times\left(\sum_{j=0}^{s}\left(H_{10} A_{j i}+K X_{1} A_{j i}-K_{j i} C\right)\right. \\
& \left.\times e\left(t-\tau_{j}(t)\right)+H_{1} F_{i} f(t)+H_{1} R_{i} d(t)\right), \\
r(t)= & T C e(t) .
\end{aligned}\right.
$$

Now, the residual signal is expressed in internal form, that is, in terms of disturbances and faults:

$$
r(t)=\sum_{i=1}^{h} \rho_{i}(\theta(t))\left(G_{r d}^{i} d(t)+G_{r f}^{i} f(t)\right),
$$

where, by abuse of notation, the following definitions are introduced:

$$
\begin{aligned}
G_{r d}^{i} & :=\left(\begin{array}{c|c}
\Lambda & H_{1} R_{i} \\
\hline T C & 0
\end{array}\right), \\
G_{r f}^{i} & :=\left(\begin{array}{c|c}
\Lambda & H_{1} F_{i} \\
\hline T C & 0
\end{array}\right),
\end{aligned}
$$

which represent the transfer from disturbance $d(t)$ and fault $f(t)$ to residual $r(t)$ in the $i$-th subsystem, respectively, where

$$
\Lambda=\sum_{j=0}^{s}\left(H_{10} A_{j i}+K X_{1} A_{j i}-K_{j i} C\right) e^{-\tau_{j} s} .
$$

This notation of Eqns. (37) and (38) was introduced here just for ease of representation, and all the following calculations will be carried out in the time domain. For attenuating the disturbance effect on the residual signal, Lemma 1 can be directly used such that the residual dynamic system is robustly stable in the presence of unknown inputs. On the other hand, to have an acceptable level of residual fault sensitivity, a reference model $W_{\text {ref }}^{i}$ is introduced for each subsystem which characterizes the residual ideal behavior in the presence of faults in the system (Mazars et al., 2008):

$$
W_{\mathrm{ref}}^{i}=\left(\begin{array}{c|c}
A_{\mathrm{ref}}^{i} & B_{\mathrm{ref}}^{i} \\
\hline C_{\mathrm{ref}}^{i} & D_{\mathrm{ref}}^{i}
\end{array}\right) .
$$

These reference models have $n_{\text {ref }}$ states, $k_{f}$ faults as input and $k_{r}$ ideal residuals as outputs, and their state space matrices are with appropriate dimensions. The reference models are chosen from stable transfer matrices with the following property:

$$
\left\|W_{\text {ref }}^{i}\right\|_{-}=\inf _{w \in \mathbb{R}}\left(\underline{\sigma}\left(W_{\text {ref }}^{i}(j w)\right)\right) \geq 1,
$$

where $\|\cdot\|_{-}$denotes the $\mathcal{H}_{-}$index and $\underline{\sigma}(\cdot)$ is the minimum singular value. The reference models with the condition (40) are suitable for fault detection because they have no fault attenuation in any frequency range (Ichalal et al., 2009). The condition (40) can be tested with the following lemma.

Lemma 2. (Mazars et al., 2008) Let the reference model be $W_{\mathrm{ref}}^{i}$ as defined in (39); the condition (40) holds if and only if there exists a symmetric matrix $P_{\mathrm{ref}} \in \mathbb{R}^{n_{\mathrm{ref}} \times n_{\mathrm{ref}}}$ such that

$$
\begin{aligned}
& {\left[\begin{array}{l}
P_{\mathrm{ref}} A_{\mathrm{ref}}^{i}+A_{\mathrm{ref}}^{i} P_{\mathrm{ref}}+C_{\mathrm{ref}}^{i} C_{\mathrm{ref}}^{i} \\
B_{\mathrm{ref}}^{i} P_{\mathrm{ref}}+D_{\mathrm{ref}}^{i} C_{\mathrm{ref}}^{i}
\end{array}\right.} \\
& \left.\begin{array}{l}
P_{\text {ref }} B_{\text {ref }}^{i}+C_{\text {ref }}^{i{ }^{T}} D_{\text {ref }}^{i} \\
D_{\text {ref }}^{i}{ }^{T} D_{\text {ref }}^{i}-I_{k_{f}}
\end{array}\right] \geq 0 .
\end{aligned}
$$

Mazars et al. (2008) present a linearization procedure to satisfy the condition (41) for reference model selection in state space systems, but provide a suboptimal solution due to its linearization procedure. In the case of time delay systems, Ahmadizadeh et al. (2014) suggested a methodology for selecting the reference model. The method of Ahmadizadeh et al. (2014) is based on satisfying a related $\mathcal{H}_{-}$index to design the reference 
model. However, this method is not applicable since it cannot be extended to our system because the term $D_{f} f(t)$ is not present in the system output equation (1). Further research may be conducted to present a method for designing an appropriate reference model for the singular delayed LPV system (1).

Now the residual error with respect to these ideal reference models is defined:

$$
e_{r}(t)=r(t)-\sum_{i=1}^{h} \rho_{i}(\theta(t)) W_{\mathrm{ref}}^{i} f(t) .
$$

With this formulation, the goal of enhancing residual fault sensitivity can be replaced by an equivalent one of attenuating the fault effect on the residual error. Then, by using Lemma1, this problem can also be addressed. Thus, the robust fault detection problem has three objectives:

1. The residual dynamic system (35) should be stable.

2. The effect of unknown inputs on the residual signal should be minimized: 1

$$
\left\|G_{r d}^{i}\right\|_{\infty}<\gamma_{d}
$$

3. The fault effect on the residual error should be minimized 2

$$
\left\|G_{e_{r} f}^{i}\right\|_{\infty}=\left\|G_{r f}^{i}-W_{\mathrm{ref}}^{i}\right\|_{\infty}<\gamma_{f} .
$$

Now, with the material provided so far, a theorem can be stated that provides the solution to the robust fault detection problem for singular delayed LPV systems considering the previous goals.

Theorem 1. Assume that, for a given scalar $a \in[0,1]$ and the reference models $W_{\mathrm{ref}}^{i}$ with the constraint (40), there exist symmetric positive definite matrices $P_{1}, P_{2}$, $Q_{1 j}, Q_{2 j}$ for $j=1, \ldots, s$, matrices $T, M$ and $M_{j i}$ for $j=0, \ldots, s$ and $i=1, \ldots, h$ and positive scalars $\bar{\gamma}_{f}$ and $\bar{\gamma}_{d}$ as the solution to the following optimization problem:

$$
\min _{P_{1}, P_{2}, Q_{1 j}, Q_{2 j}, M, M_{j i}, T, \bar{\gamma}_{d}, \bar{\gamma}_{f}} a \bar{\gamma}_{f}+(1-a) \bar{\gamma}_{d}
$$

subject to the LMI constraints

$$
\left[\begin{array}{cccccc}
\Omega_{0}^{i} & \Omega_{1}^{i} & \cdots & \Omega_{s}^{i} & \bar{R}_{i} & C^{T} T^{T} \\
* & \bar{Q}_{11} & \cdots & 0 & 0 & 0 \\
\vdots & \vdots & \ddots & \vdots & \vdots & \vdots \\
* & * & \cdots & \bar{Q}_{1 s} & 0 & 0 \\
* & * & \cdots & * & -\bar{\gamma}_{d} I & 0 \\
* & * & \cdots & * & * & -I
\end{array}\right]<0
$$

\footnotetext{
${ }^{1}$ This criterion is formulated as $\|r(t)\|_{2}<\gamma_{d}\|d(t)\|_{2}$ and, by abuse of notation, written as (43).

${ }^{2}$ This criterion is formulated as $\left\|e_{r}(t)\right\|_{2}<\gamma_{f}\|f(t)\|_{2}$ and, by abuse of notation, written as (44).
}

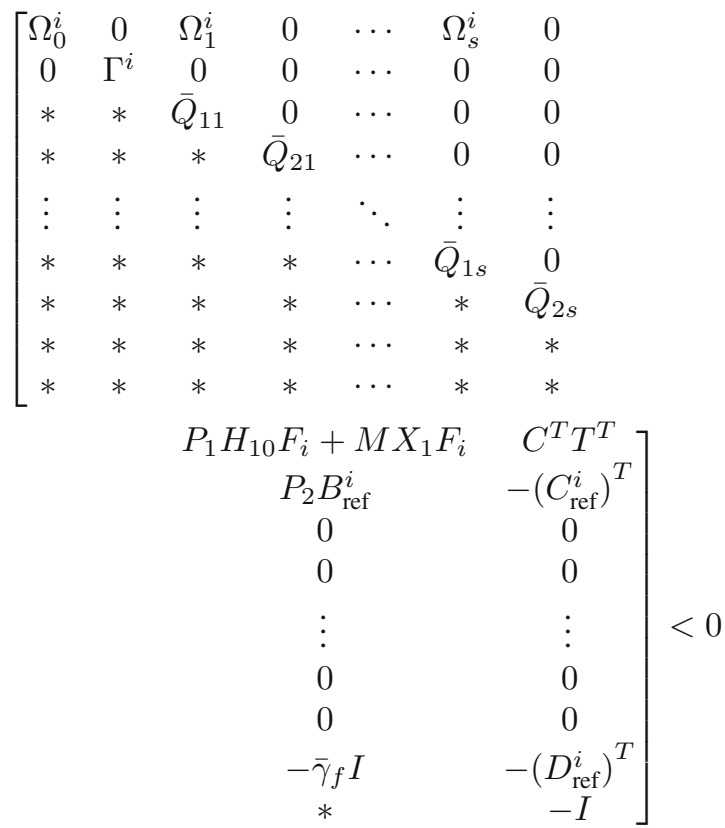

for all $i=1, \ldots, h$, and where for $i=1, \ldots, h$ and $j=$ $1, \ldots, s$ we have

$$
\begin{aligned}
\Omega_{0}^{i}= & P_{1} H_{10} A_{0 i}+M X_{1} A_{0 i}-M_{0 i} C \\
& +\left(P_{1} H_{10} A_{0 i}+M X_{1} A_{0 i}-M_{0 i} C\right)^{T}+Q_{11} \\
& +\cdots+Q_{1 s}, \\
\Omega_{j}^{i}= & P_{1} H_{10} A_{j i}+M X_{1} A_{j i}-M_{j i} C, \\
\Gamma^{i}= & P_{2} A_{\mathrm{ref}}^{i}+\left(P_{2} A_{r e f}^{i}\right)^{T}+Q_{21}+\cdots+Q_{2 s}, \\
\bar{Q}_{1 j}= & -\left(1-\mu_{j}\right) Q_{1 j}, \\
\bar{Q}_{2 j}= & -\left(1-\mu_{j}\right) Q_{2 j}, \\
\bar{R}_{i}= & P_{1} H_{10} R_{i}+M X_{1} R_{i} .
\end{aligned}
$$

Then the robust residual generator (6) exists and can be determined as follows:

$$
\begin{aligned}
K & =P_{1}^{-1} M, \\
K_{j i} & =P_{1}^{-1} M_{j i} .
\end{aligned}
$$

The observer matrices $N_{j i}, L_{j i}, G_{i}$ and $H_{2}$ can be calculated from (33), (34), (16) and (30), with attenuation levels regarding unknown inputs on residuals and faults on residual errors given respectively by

$$
\begin{aligned}
\gamma_{d} & =\sqrt{\bar{\gamma}_{d}} \\
\gamma_{f} & =\sqrt{\bar{\gamma}_{f}} .
\end{aligned}
$$

Proof. With the notation introduced in (38), the $i$-th transfer matrix from the fault to the residual error is

$$
\begin{aligned}
G_{r f}^{i} & -W_{\mathrm{ref}}^{i} \\
& =\left(\begin{array}{cc|c}
\Lambda & 0 & H_{10} F_{i}+K X_{1} F_{i} \\
0 & A_{\mathrm{ref}}^{i} & B_{\mathrm{ref}}^{i} \\
\hline T C & -C_{\mathrm{ref}}^{i} & -D_{\mathrm{ref}}^{i}
\end{array}\right) .
\end{aligned}
$$


Choosing symmetric positive definite block diagonal matrices

$$
P=\left[\begin{array}{cc}
P_{1} & 0 \\
0 & P_{2}
\end{array}\right]
$$

and

$$
Q_{j}=\left[\begin{array}{cc}
Q_{1 j} & 0 \\
0 & Q_{2 j}
\end{array}\right]
$$

the attenuation of the fault effect on residual error can be addressed by using Lemma1 1 Substituting the associated terms in 19) results in

$$
\begin{aligned}
& {\left[\begin{array}{ccccccc}
\bar{\Omega}_{0}^{i} & 0 & \bar{\Omega}_{1}^{i} & 0 & \ldots & \bar{\Omega}_{s}^{i} & 0 \\
0 & \Gamma^{i} & 0 & 0 & \cdots & 0 & 0 \\
* & * & \bar{Q}_{11} & 0 & \ldots & 0 & 0 \\
* & * & * & \bar{Q}_{21} & \cdots & 0 & 0 \\
\vdots & \vdots & \vdots & \vdots & \ddots & \vdots & \vdots \\
* & * & * & * & \cdots & \bar{Q}_{1 s} & 0 \\
* & * & * & * & \cdots & * & \bar{Q}_{2 s} \\
* & * & * & * & \cdots & * & * \\
* & * & * & * & \ldots & * & *
\end{array}\right.} \\
& P_{1} H_{10} F_{i}+P_{1} K X_{1} F_{i} \quad C^{T} T^{T} \\
& P_{2} B_{\text {ref }}^{i} \\
& 0 \\
& 0 \\
& \text { : } \\
& 0 \\
& 0 \\
& -\gamma_{f}^{2} I \\
& \left.\begin{array}{c}
-\left(C_{\mathrm{ref}}^{i}\right)^{T} \\
0 \\
0 \\
\vdots \\
0 \\
0 \\
-\left(D_{\mathrm{ref}}^{i}\right)^{T} \\
-I
\end{array}\right]<0
\end{aligned}
$$

where for $i=1, \ldots, h$ and $j=1, \ldots, s$

$$
\begin{aligned}
\bar{\Omega}_{0}^{i}= & P_{1} H_{10} A_{0 i}+P_{1} K X_{1} A_{0 i}-P_{1} K_{0 i} C \\
& +\left(P_{1} H_{10} A_{0 i}+P_{1} K X_{1} A_{0 i}-P_{1} K_{0 i} C\right)^{T} \\
& +Q_{11}+\cdots+Q_{1 s}, \\
\bar{\Omega}_{j}^{i}= & P_{1} H_{10} A_{j i}+P_{1} K X_{1} A_{j i}-P_{1} K_{j i} C, \\
\Gamma^{i}= & P_{2} A_{\mathrm{ref}}^{i}+\left(P_{2} A_{\mathrm{ref}}^{i}\right)^{T}+Q_{21}+\cdots+Q_{2 s}, \\
\bar{Q}_{1 j}= & -\left(1-\mu_{j}\right) Q_{1 j}, \\
\bar{Q}_{2 j}= & -\left(1-\mu_{j}\right) Q_{2 j} .
\end{aligned}
$$

Obviously, this is a nonlinear matrix inequality because of product terms of unknown variables, so the following change of variables is applied:

$$
\begin{aligned}
M & :=P_{1} K, \\
M_{j i} & :=P_{1} K_{j i}, \\
\bar{\gamma}_{f} & :=\gamma_{f}^{2} .
\end{aligned}
$$

Then the LMI 47) is obtained. According to Lemma 1 disturbance attenuation in the residual system (36) can be addressed in a similar manner. By using $P_{1}$ and $Q_{1 j}(j=1, \ldots, s)$ as positive definite matrices for $P$ and $Q_{j}$ in Lemma 1 and substituting the associated expressions of matrices of the transfer matrix $G_{r d}^{i}$ in (19) and again by using the change of variables (54) -55 , and

$$
\bar{\gamma}_{d}:=\gamma_{d}^{2}
$$

the nonlinearity in the matrix inequality is resolved and the LMI (46) is obtained. Moreover, 48 - 51 can be directly derived from (54) -57). Finally, the simultaneous problem of robustness to disturbances and sensitivity to faults can be addressed by the multi-objective optimization problem (45) with the constraints (46) and (47) considering that the parameter $a \in[0,1]$ is used to adjust the objective weighting.

Summary of the method. A summary of the presented method for robust fault detection in singular LPV systems with multiple time varying delays is presented in Algorithm 1

$\overline{\text { Algorithm 1. Robust fault detection system design for a }}$ singular LPV system with multiple time varying delays.

Step 1. Check Assumption 2.

Step 2. Calculate $H_{10}$ and $X_{1}$ from the first $n$ columns of $\left[\begin{array}{l}E \\ C\end{array}\right]^{+}$and $X=I_{n+m}-\left[\begin{array}{l}E \\ C\end{array}\right]\left[\begin{array}{l}E \\ C\end{array}\right]^{+}$, respectively.

Step 3. Choose some appropriate reference models $W_{\text {ref }}^{i}$ satisfying the constraint (40) by checking Lemma2.

Step 4. Solve the optimization problem (45) under the LMI constraints (46), and 47) and obtain matrices $P_{1}$, $P_{2}, M, T, Q_{1 j}, Q_{2 j}$ for $j=1, \ldots, s$ and $M_{j i}$ for $j=$ $0, \ldots, s$ and $i=1, \ldots, h$.

Step 5. Calculate $K$ and $K_{j i}$ for $j=0, \ldots, s$ and $i=$ $1, \ldots, h$ from (48) and 49), respectively.

Step 6. Calculate $\mathrm{H}_{2}, G$ and $\mathrm{N}_{j i}$ for $j=0, \ldots, s$ and $i=1, \ldots, h$ from (30), 16) and (33), respectively.

Step 7. Calculate $L_{j i}$ for $j=0, \ldots, s$ and $i=1, \ldots, h$ from (34).

\section{Application}

5.1. Description. In order to show the performance of the proposed fault detection approach, a case study based on the Riera Blanca catchment of the Barcelona sewer network is considered. This catchment has already been used for illustrating the model predictive control of sewer networks in the work of Puig et al. (2009). This catchment can be modeled using the virtual tank approach 
described by Ocampo Martínez et al. (2013), leading to the representation shown in Fig. 1 .

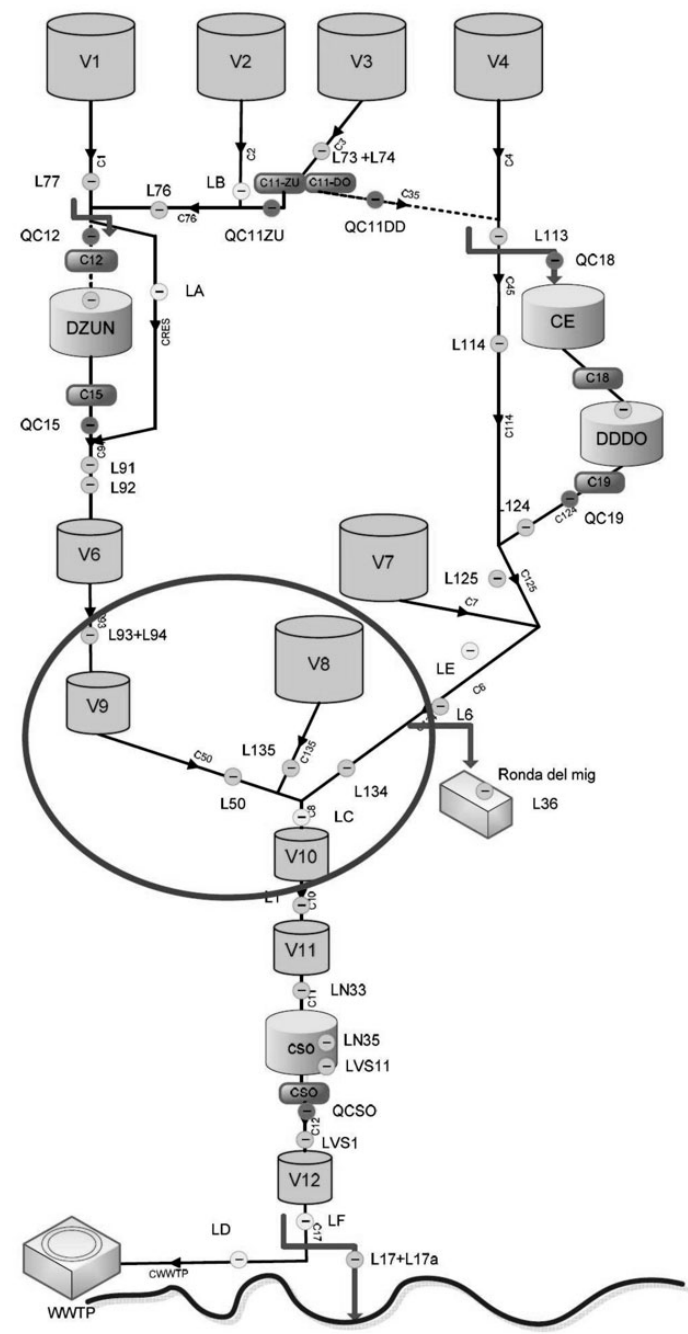

Fig. 1. Riera Blanca subnetwork representation using the virtual tank modeling approach.

The part of the Riera Blanca catchment considered in this paper is highlighted in Fig. 1 The equations corresponding to this part are the following:

$$
\begin{gathered}
\left\{\begin{array}{l}
\dot{v}_{8}(t)=-c v c_{8}(t) v_{8}(t)+\varepsilon_{8} S_{8} P_{19}(t-\tau), \\
q_{8}(t)=c_{8} v_{8}(t),
\end{array}\right. \\
\left\{\begin{array}{c}
\dot{v}_{9}(t)=-c v c_{9}(t) v_{9}(t)+\varepsilon_{9} S_{9} P_{16}(t-\tau) \\
+C_{93}(t)+f(t), \\
q_{9}(t)=c_{9} v_{9}(t),
\end{array}\right.
\end{gathered}
$$

$$
\left\{\begin{aligned}
\dot{v}_{10}(t)= & c v c_{8}(t) v_{8}(t-\tau)+c v c_{9}(t) v_{9}(t-\tau) \\
& -c v c_{10}(t) v_{10}(t)+\varepsilon_{10} S_{10} P_{16}(t-\tau) \\
& +C_{134}(t)+d(t), \\
q_{10}(t)= & c_{10} v_{10}(t),
\end{aligned}\right.
$$

where $v_{i}(t)(i=8,9,10)$ are water volumes stored in catchment $i$ (virtual tank $i$ ) and $q_{i}(t)(i=8,9,10)$ are the output flows from these catchments. Here $\varepsilon_{i}$ and $S_{i}(i=8,9,10)$ are absorption coefficients and the surfaces of these catchments, respectively. $P_{19}(t)$ is the rain intensity falling in catchment 8 and $P_{16}(t)$ is the rain intensity falling in catchments 9 and $10 . C_{93}(t)$ is the input flow from other catchments to catchment 9 and $C_{134}(t)$ is the input flow from other catchments to catchment 10. Furthermore, $c v c_{i}(t)(i=8,9,10)$ is a volume-flow conversion coefficient for the catchment and $c_{i}(i=8,9,10)$ is the related gain of a flowmeter transducer measuring the outflow of catchment $i ; f(t)$ is a fault related to the gate in the input of catchment 9 and $d(t)$ is disturbance in the system related to upstream flows not measured; $\tau$ is the transport delay associated with the rain-fall and run-off processes. Since parameters $c v c_{i}(t)$ vary with the output flows $q_{i}(t)$, the system is a quasi-LPV one and, because of the transport delays, with delay. In order to use the methodology presented in this paper for state delayed systems, the delayed inputs are chosen as additional states for the system such that

$$
x(t)=\left[\begin{array}{lllll}
v_{8}(t) & v_{9}(t) & v_{10}(t) & P_{19}(t) & P_{16}(t)
\end{array}\right]^{T} .
$$

On the other hand, the vector of system inputs is

$$
u(t)=\left[\begin{array}{llll}
P_{19}(t) & P_{16}(t) & C_{93}(t) & C_{134}(t)
\end{array}\right]^{T},
$$

while the vector of system outputs is

$$
y(t)=\left[\begin{array}{llll}
q_{8}(t) & q_{9}(t) & P_{19}(t) & P_{16}(t)
\end{array}\right]^{T} .
$$

Thus, Eqns. (58) - 60) can be brought to the form

$$
\left\{\begin{aligned}
E \dot{x}(t)= & A_{0}(\theta(t)) x(t)+A_{1}(\theta(t)) x(t-\tau) \\
& +B u(t)+R d(t)+F f(t), \\
y(t)= & C x(t)
\end{aligned}\right.
$$

where

$$
E=\left[\begin{array}{lllll}
1 & 0 & 0 & 0 & 0 \\
0 & 1 & 0 & 0 & 0 \\
0 & 0 & 1 & 0 & 0 \\
0 & 0 & 0 & 0 & 0 \\
0 & 0 & 0 & 0 & 0
\end{array}\right]
$$

$$
\begin{aligned}
& A_{0}(\theta(t)) \\
& =\left[\begin{array}{ccccc}
-c v c_{8}(t) & 0 & 0 & 0 & 0 \\
0 & -c v c_{9}(t) & 0 & 0 & 0 \\
0 & 0 & -c v c_{10}(t) & 0 & 0 \\
0 & 0 & 0 & -1 & 0 \\
0 & 0 & 0 & 0 & -1
\end{array}\right]
\end{aligned}
$$




$$
\begin{gathered}
A_{1}(\theta(t))=\left[\begin{array}{ccccc}
0 & 0 & 0 & \varepsilon_{8} S_{8} & 0 \\
0 & 0 & 0 & 0 & \varepsilon_{9} S_{9} \\
c v c_{8}(t) & c v c_{9}(t) & 0 & 0 & \varepsilon_{10} S_{10} \\
0 & 0 & 0 & 0 & 0 \\
0 & 0 & 0 & 0 & 0
\end{array}\right], \\
B=\left[\begin{array}{llll}
0 & 0 & 0 & 0 \\
0 & 0 & 1 & 0 \\
0 & 0 & 0 & 1 \\
1 & 0 & 0 & 0 \\
0 & 1 & 0 & 0
\end{array}\right], \quad R=\left[\begin{array}{l}
0 \\
0 \\
1 \\
0 \\
0
\end{array}\right], \\
F=\left[\begin{array}{l}
0 \\
1 \\
0 \\
0 \\
0
\end{array}\right], \quad C=\left[\begin{array}{ccccc}
c_{8} & 0 & 0 & 0 & 0 \\
0 & c_{9} & 0 & 0 & 0 \\
0 & 0 & 0 & 1 & 0 \\
0 & 0 & 0 & 0 & 1
\end{array}\right],
\end{gathered}
$$

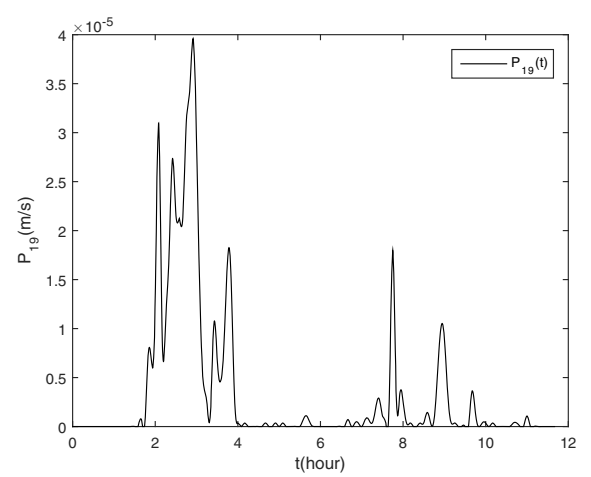

(a)

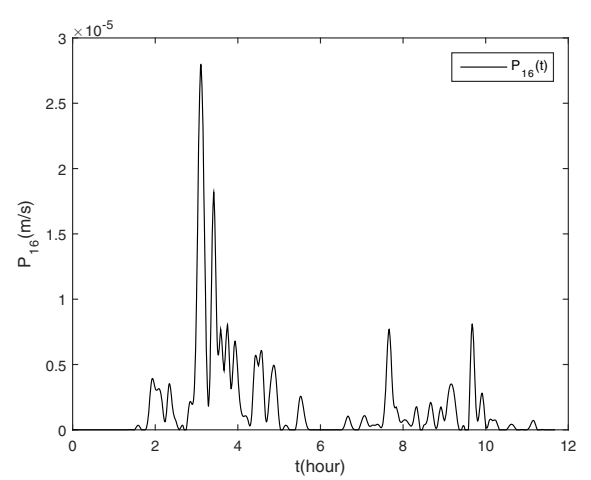

(b)

\begin{tabular}{|c|c|c|c|}
\hline$i$ & $\mathbf{8}$ & $\mathbf{9}$ & $\mathbf{1 0}$ \\
\hline \hline$\varepsilon_{i}$ & 0.584383 & 0.048593 & 0.9855 \\
\hline
\end{tabular}

Table 2. Surface of catchment $i$.

\begin{tabular}{|l|c|c|c|}
\hline$i$ & $\mathbf{8}$ & $\mathbf{9}$ & $\mathbf{1 0}$ \\
\hline \hline$S_{i}\left(m^{2}\right)$ & 769800 & 722500 & 149000 \\
\hline
\end{tabular}

Table 3. Gain of flowmeter transducer of catchment $i$.

\begin{tabular}{|l|c|c|c|}
\hline$i$ & $\mathbf{8}$ & $\mathbf{9}$ & $\mathbf{1 0}$ \\
\hline \hline$c_{i}(1 / s)$ & 0.003 & 0.0026 & 0.003 \\
\hline
\end{tabular}

A real scenario of one day raining has been used as input data. In particular, the amount of water coming from other catchments, i.e., $C_{93}(t)$ and $C_{134}(t)$, measured from the available flowmeters as well as the amount of rain entering to the catchments considered, i.e., $P_{16}(t)$ and $P_{19}(t)$, reading from rain gauges have been used as the system inputs. These inputs have been provided in 5-minute time samples and are depicted in Fig. 2.

In the system under consideration, there are three LPV parameters that correspond to the volume-flow conversion coefficients $c v c_{i}(t)$, which are varying with

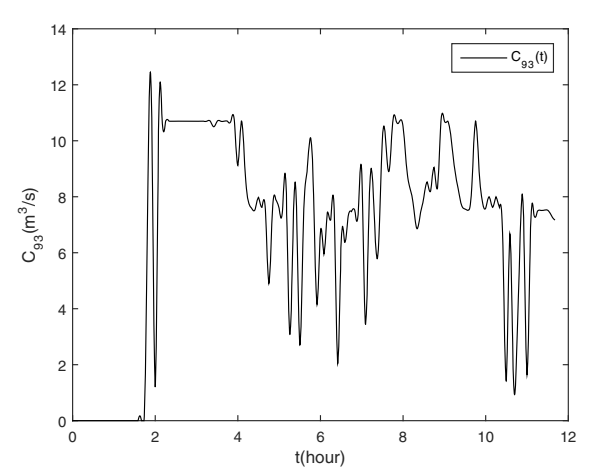

(c)

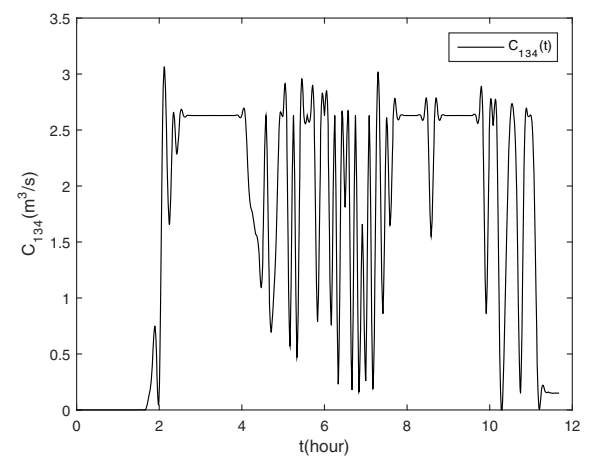

(d)

Fig. 2. System inputs: $P_{19}(t)$ (a), $P_{16}(t)$ (b), $C_{93}(t)$ (c), $C_{134}(t)(d)$. 


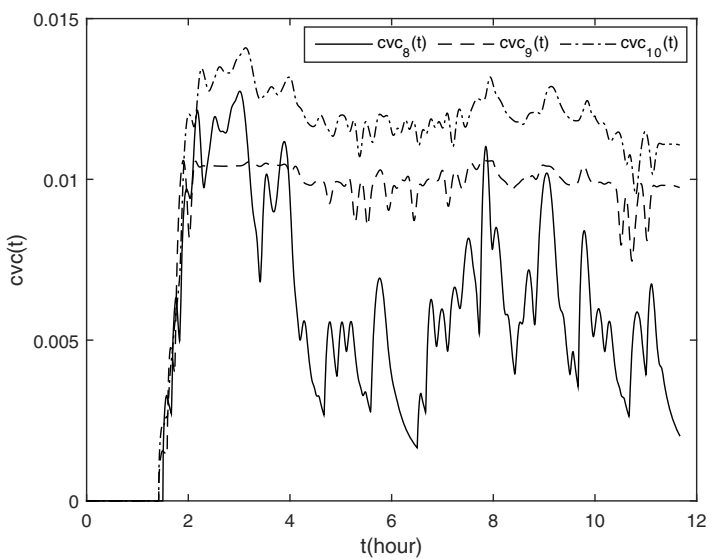

Fig. 3. Evolution of LPV parameters.

the operating point and can be scheduled in real-time. The time evolution of system parameters for the scenario considered is depicted in Fig. 3. Since there are three parameters, eight subsystems are defined, each of them at the vertices of the parameter space. The weights of the different subsystems are calculated based on the following formula:

$$
\begin{aligned}
& \rho_{i}(t) \\
& =\left\{\prod_{k=1}^{3}\left(i_{k}+(-1)^{i_{k}} \alpha_{k}(t)\right) \mid i_{k}=0,1\right. \\
& \left.\quad \text { for } k=1,2,3 \text { and } i=4 i_{1}+2 i_{2}+i_{3}+1\right\},
\end{aligned}
$$

where

$$
\alpha_{k}(t)=\frac{\theta_{k}^{M}-\theta_{k}(t)}{\theta_{k}^{M}-\theta_{k}^{m}},
$$

in which $\theta_{k}(t)$ for $k=1,2,3$ are three LPV system parameters and $\rho_{i}(t)$ for $i=1,2, \ldots, 8$ are the weights associated with each subsystem in the polytopic model. Here $\theta_{k}^{m}$ and $\theta_{k}^{M}$ are respectively, minimum and maximum values of the range of variation of $\theta_{k}(t)$.

The weighting function 61) is formulated for the three-parameter case, but can easily be extended and used for systems with any number of parameters. The evolution of eight subsystem weights is depicted in Fig. 4

The matrices in the $i$-th vertex of the parameters domain can be constructed with the following formula:

$A_{j i}$

$$
\begin{gathered}
=\left\{A_{j}^{0}+\left.\Delta A_{j}\right|_{\theta_{k}=(-1)^{i_{k}+1} \Delta \theta_{k} \text { for } k=1,2,3}, i_{k}=0,1\right. \\
\text { for } \left.k=1,2,3 \text { and } i=4 i_{1}+2 i_{2}+i_{3}+1\right\},
\end{gathered}
$$

where

$$
A_{j}^{0}=\left.A_{j}(\theta(t))\right|_{\theta_{k}=\bar{\theta}_{k} \text { for } k=1,2,3,}
$$

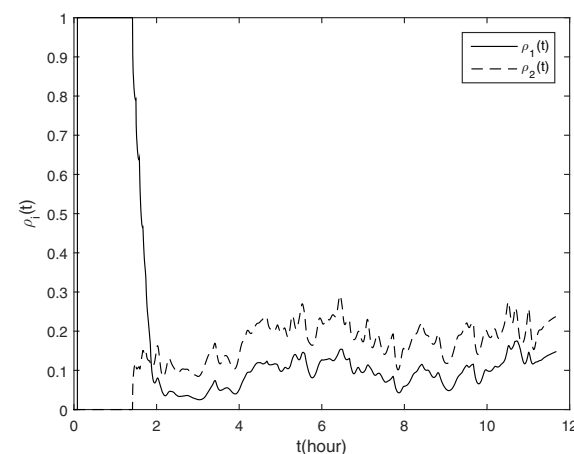

(a)

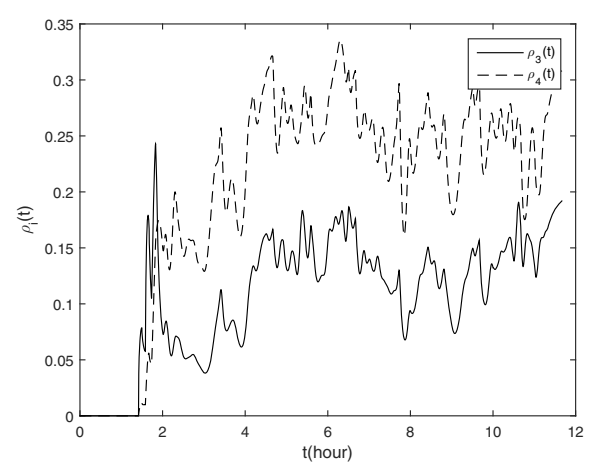

(b)

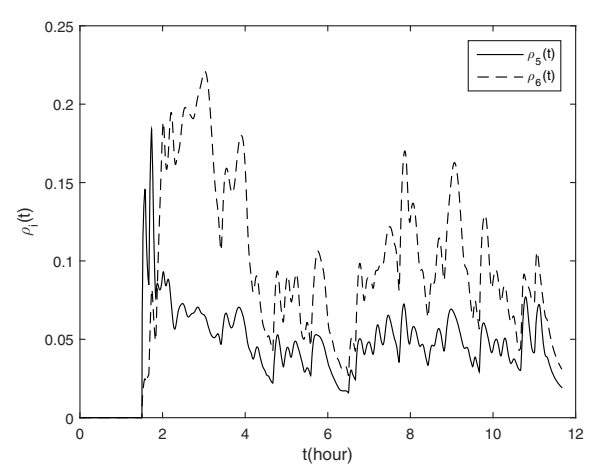

(c)

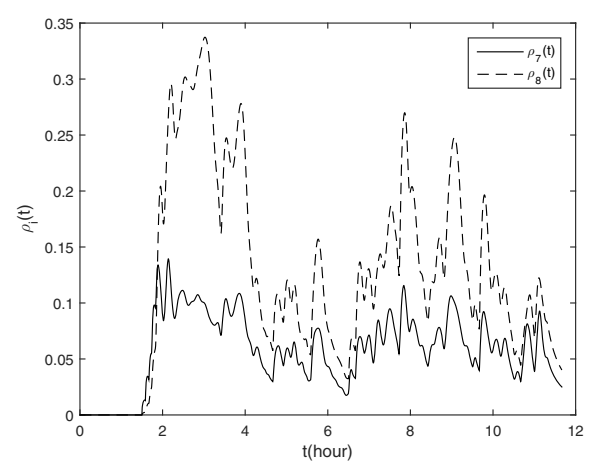

(d)

Fig. 4. Evolution of subsystem weights: $\rho_{1}(t)$ and $\rho_{2}(t)$ (a), $\rho_{3}(t)$ and $\rho_{4}(t)(\mathrm{b}), \rho_{5}(t)$ and $\rho_{6}(t)(\mathrm{c}), \rho_{7}(t)$ and $\rho_{8}(t)$ (d), 


$$
\Delta A_{j}(\theta(t))=A_{j}(\theta(t))-\left(\left.A_{j}(\theta(t))\right|_{\theta_{k}=0 \text { for } k=1,2,3}\right),
$$

in which $j=0,1$, while the central value and the amount of maximum variation around this central value related to $\theta_{k}(t)$ for $k=1,2,3$ are given by

$$
\begin{gathered}
\bar{\theta}_{k}=\frac{1}{2}\left(\theta_{k}^{m}+\theta_{k}^{M}\right), \\
\Delta \theta_{k}=\frac{1}{2}\left(\theta_{k}^{M}-\theta_{k}^{m}\right) .
\end{gathered}
$$

5.2. Results. The UIO proposed in this paper is designed according to Algorithm 1 for the following reference model for all subsystems

$$
W_{\text {ref }}=1+\frac{1}{s+1},
$$

which satisfies (40). This reference model forces the residual to have more sensitivity at low frequencies while having minimum sensitivity with a unity gain in other frequency ranges. The state space realization of $W_{\text {ref }}$ is

$$
W_{\text {ref }}=\left(\begin{array}{c|c}
-1 & 1 \\
\hline 1 & 1
\end{array}\right) .
$$

The adjusting parameter $a$ in 45 is chosen to be $a=0.5$ to have equal weights on disturbance rejection and fault sensitivity. The optimization problem (45) with the LMI constraints (46) and (47) is solved with the Sedumi solver (Sturm, 1999) via the YALMIP toolbox (Lofberg, 2004).

The simulations show that the optimization problem (45) subject to constraints (46) and (47) tends to decrease the norm of the matrix $T$. According to the internal form of the residual (35), the matrix $T$ has the same effect on the norm of $G_{r f}$ and $G_{r d}$, and it adjusts the steady state response of the residual. To avoid a small residual, its norm is imposed to be greater than one. The Lyapunov-Krasovskii functional matrices are

$$
\begin{gathered}
P_{1}=\left[\begin{array}{ccc}
1.6300 & -0.0000 & -0.0000 \\
-0.0000 & 1.6301 & 0.0000 \\
-0.0000 & 0.0000 & 0.0000 \\
0.0025 & -0.0002 & 0.0000 \\
-0.0014 & -0.0001 & -0.0000 \\
0.0025 & -0.0014 \\
-0.0002 & -0.0001 \\
0.0000 & -0.0000 \\
1.6304 & -0.0000 \\
-0.0000 & 1.6304
\end{array}\right] \\
P_{2}=2.0000
\end{gathered}
$$

$$
\begin{aligned}
Q_{11}= & {\left[\begin{array}{ccc}
1.6304 & 0.0000 & 0.0000 \\
0.0000 & 1.6303 & -0.0000 \\
0.0000 & -0.0000 & 0.0000 \\
-0.0000 & 0.0000 & 0.0000 \\
0.0000 & -0.0000 & 0.0000 \\
-0.0000 & 0.0000 \\
0.0000 & -0.0000 \\
0.0000 & 0.0000 \\
1.6304 & 0.0000 \\
& -0.0000 & 1.6304
\end{array}\right] } \\
& Q_{21}=1.6018 \times 10^{-10} .
\end{aligned}
$$

The values of $K$ and $T$ obtained are

$$
\begin{aligned}
& K=10^{6} \times\left[\begin{array}{cccc}
-0.0000 & 0.0009 & 0 & 0.0000 \\
0.0000 & -0.1511 & 0 & -0.0000 \\
-0.0000 & 4.6080 & 0 & 0.0000 \\
0.0000 & 0.0550 & 0 & -0.0000 \\
-0.0000 & 0.0550 & 0 & -0.0000
\end{array}\right. \\
& \left.\begin{array}{lllll}
-0.0000 & -0.0000 & 0.0000 & 0 & 0
\end{array}\right] \\
& \begin{array}{lllll}
-0.0000 & 0.0000 & -0.0000 & 0 & 0
\end{array} \\
& \begin{array}{lllll}
-0.0000 & -0.0000 & 0.0081 & 0 & 0
\end{array} \\
& \begin{array}{lllll}
-0.0000 & 0.0000 & 0.0000 & 0 & 0
\end{array} \\
& \left.\begin{array}{lllll}
-0.0000 & -0.0000 & 0.0000 & 0 & 0
\end{array}\right] \\
& T=\left[\begin{array}{llll}
1.6301 & 1.6300 & 0.3013 & 0.3013
\end{array}\right] .
\end{aligned}
$$

The results obtained for $G$ and $H_{2}$ are

$$
\begin{gathered}
G=\left[\begin{array}{cccc}
0.0000 & -0.0000 & 0.0057 & 0 \\
-0.0000 & -0.0000 & 0.0053 & 0 \\
1.5490 & -0.5708 & 9.9850 & 1.0000 \\
-0.0197 & -0.0000 & 0.3696 & 0 \\
-0.0000 & -0.0202 & 0.3696 & 0
\end{array}\right], \\
H_{2}=10^{3} \times\left[\begin{array}{cccc}
0.0000 & -0.0022 & 0 & 0 \\
0.0000 & 0.3826 & 0 & 0 \\
-0.0001 & -3.8404 & 0 & 0 \\
0.0000 & -0.1421 & 0.0010 & 0 \\
-0.0000 & -0.1421 & 0 & 0.0010
\end{array}\right] .
\end{gathered}
$$

Although there are eight subsystems, due to space limitations, only the resulting matrices for the first subsystem are presented here:

$$
\begin{aligned}
K_{01}=10^{6} \times\left[\begin{array}{cccc}
0.0003 & -0.0000 & -0.0000 & 0.0000 \\
-0.0000 & 0.0004 & 0.0000 & 0.0000 \\
1.7460 & -0.2861 & -0.0001 & 0.0000 \\
-0.0000 & -0.0000 & 0.0000 & 0.0000 \\
-0.0000 & -0.0000 & -0.0000 & 0.0000
\end{array}\right], \\
K_{11}=10^{5} \times\left[\begin{array}{cccc}
0.0000 & -0.0000 & 4.4985 & 0.0020 \\
-0.0000 & 0.0000 & 0.0000 & 0.0018 \\
0.0000 & -0.0000 & 0.0008 & 4.9740 \\
-0.0000 & 0.0000 & 0.0000 & 0.1297 \\
0.0000 & -0.0000 & 0.0000 & 0.1298
\end{array}\right],
\end{aligned}
$$




$$
N_{01}=10^{3} \times\left[\begin{array}{ccc}
-0.0010 & 0.0000 & 0 \\
0.0000 & -0.0010 & 0 \\
-5.2380 & 0.7438 & 0 \\
0.0000 & 0.0000 & 0 \\
0.0000 & 0.0000 & 0 \\
0.0000 & -0.0000 \\
-0.0000 & -0.0000 \\
0.0714 & -0.0315 \\
-0.0010 & -0.0000 \\
0.0001 & -0.0010
\end{array}\right],
$$

$N_{11}=\left[\begin{array}{ccccc}-0.0000 & 0.0000 & 0 & -0.0000 & 0.0000 \\ 0.0000 & -0.0000 & 0 & -0.0000 & 0.0000 \\ -0.0014 & 0.0040 & 0 & -0.0317 & 0.0636 \\ 0.0000 & -0.0000 & 0 & -0.0000 & 0.0000 \\ -0.0000 & 0.0000 & 0 & -0.0000 & -0.0000\end{array}\right]$

$L_{01}=10^{6} \times\left[\begin{array}{cccc}0.0003 & 0.0000 & -0.0000 & 0.0000 \\ -0.0000 & 0.0000 & 0.0000 & 0.0000 \\ 1.7460 & 0.0044 & -0.0000 & 0.0000 \\ -0.0000 & 0.0001 & 0.0000 & 0.0000 \\ -0.0000 & 0.0001 & 0.0000 & 0.0000\end{array}\right]$

$L_{11}=10^{5} \times\left[\begin{array}{cccc}0.0000 & 0.0000 & 4.4985 & 0.0020 \\ -0.0000 & 0.0000 & -0.0000 & 0.0018 \\ 0.0000 & -0.0000 & 0.0008 & 4.9740 \\ -0.0000 & 0.0000 & -0.0000 & 0.1297 \\ 0.0000 & 0.0000 & 0.0000 & 0.1298\end{array}\right]$.

Once the UIO has been designed, two fault scenarios are considered. In the first one, an abrupt fault occurring in the time period of $6-8 \mathrm{~h}$ is considered. In this scenario, in the interval of $4-10 \mathrm{~h}$, the system is affected by an offset-like disturbance. From the residual response in Fig. 5. it can be seen that the fault is detected successfully while the disturbance is rejected. It can also be noticed that the residual is almost zero for non-faulty periods, including the period in which disturbance occurs in the system. In the second scenario, fault detection has been tested with the same conditions as the first one, just replacing the abrupt fault with an incipient one. The residual is depicted now in Fig. 6. It shows that it is able to detect the fault while it is not affected by the disturbance. Moreover, it can be noticed that the residual increases according to the fault growth in the system.

\section{Conclusions}

In this paper, the problem of fault detection in singular LPV systems with multiple time-varying delays was considered. A UIO was designed for the purpose of state and output estimation in the presence of disturbances and

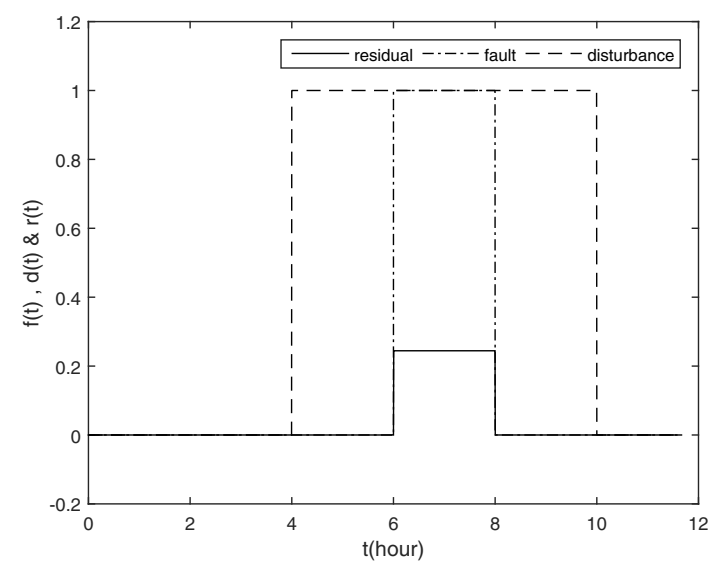

Fig. 5. Residual in the case of an abrupt fault.

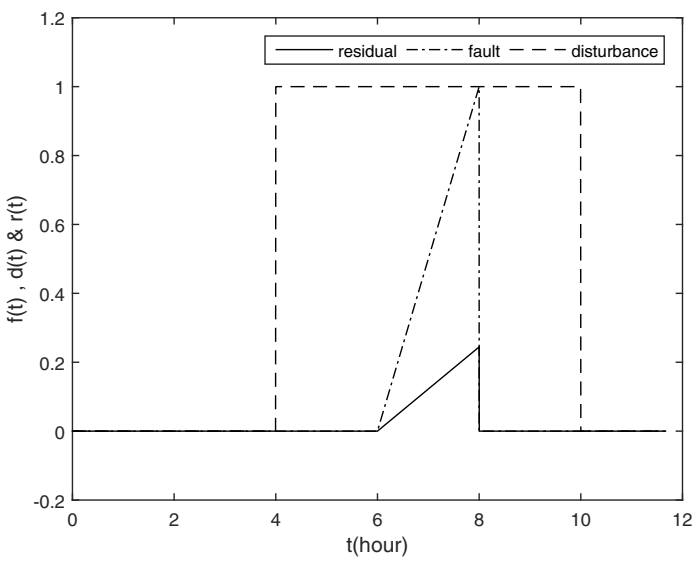

Fig. 6. Residual in the case of an incipient fault.

actuator faults. The effect of disturbance was attenuated by using a BRL for delayed LPV systems. Thus, in a non-faulty case, the UIO yields good state and output estimation. In a faulty situation, as the residual was model matched with an ideal fault-residual reference one, fault sensitivity was designed to be much greater than the disturbance effect on the residual. Thus, the fault could be detected successfully from the difference between the measurements and the estimates of output signals computed in the residual signal. This issue was formulated as a convex optimization problem with two LMI constraints, which realizes disturbance attenuation and fault sensitivity goals for the robust fault detection problem. Finally, a part of the Barcelona sewer network was used as a case study.

Successful results of this real application show the applicability of the proposed methodology. Reducing the conservatism of the conditions proposed in this paper for fault diagnosis in singular delayed LPV systems by using either a parameter dependent Lyapunov-Krasovskii functional or a delay dependent approach is one of 
the future research directions. Developing a systematic approach for designing the reference model needed in the model matching procedure for singular delayed LPV systems is another interesting topic that can be addressed in future works. This is important because, without a good reference model, robustness and fault sensitivity specifications in a fault diagnosis problem cannot be satisfied simultaneously.

\section{Acknowledgment}

The authors would like to thank the anonymous referees for their valuable comments and suggestions, which helped to improve the paper. This work was funded by the Spanish Ministry of Science and Technology and FEDER through the project CICYT HARCRICS (Ref. DPI2014-58104-R).

\section{References}

Ahmadizadeh, S., Zarei, J. and Karimi, H.R. (2014). Robust unknown input observer design for linear uncertain time delay systems with application to fault detection, Asian Journal of Control 16(4): 1006-1019.

Aouaouda, S., Chadli, M., Shi, P. and Karimi, H.R. (2015). Discrete time $H_{-} / H_{\infty}$ sensor fault detection observer design for nonlinear systems with parameter uncertainty, International Journal of Robust and Nonlinear Control 25(3): 339-361.

Bai, L., Tian, Z. and Shi, S. (2006). Design of robust $H_{\infty}$ fault detection filter for linear uncertain time delay systems, ISA Transactions 45(4): 491-502.

Bokor, J. and Balas, G. (2004). Detection filter design for LPV systems-a geometric approach, Automatica 40(3): 511-518.

Bolea, Y., Puig, V. and Blesa, J. (2014). Linear parameter varying modeling and identification for real-time control of open-flow irrigation canals, Environmental Modeling and Software 53: 87-97.

Chen, J. and Patton, R. (1999). Robust Model-Based Fault Diagnosis for Dynamic Systems, Kluwer Academic Publishers, Boston, MA.

Chen, L., Zhong, M.Y. and Zhang, M.Y. (2011). $H_{\infty}$ fault detection for linear singular systems with time-varying delay, International Journal of Control, Automation and Systems 9(1): 9-14.

Dai, L. (1989). Singular Control Systems, Springer-Verlag, Berlin.

Darouach, M. and Boutayeb, M. (1995). Design of observers for descriptor systems, IEEE Transactions on Automatic Control 40(7): 1323-1327.

Ding, S.X. (2008). Model-based Fault Diagnosis Techniques, Springer, Berlin.

Duan, G.R. (2010). Analysis and Design of Descriptor Linear Systems, Springer, New York, NY.
Duan, G.R., Howe, D. and Patton, R.J. (2002). Robust fault detection in descriptor linear systems via generalized unknown input observers, International Journal of Systems Science 33(5): 369-377.

Fattouh, A., Sename, O. and Jean-Michel, D. (1999). An unknown input observer design for linear time-delay systems, Proceedings of the 38th Conference on Decision and Control, Phoenix, AZ, USA, Vol. 4, pp. 4222-4227.

Frisk, E. and Nielsen, L. (2006). Robust residual generation for diagnosis including a reference model for residual behavior, Automatica 42(3): 437-445.

Fu, Y.M., Duan, G.R. and Song, S.M. (2004). Design of unknown input observer for linear time-delay systems, International Journal of Control, Automation, and Systems 2(4): 530-535.

Guan, Y. and Saif, M. (1991). A novel approach to the design of unknown input observers, IEEE Transactions on Automatic Control 36(5): 632-635.

Habib, H., Rodrigues, M., Mechmeche, C. and Braiek, N.B. (2010). Robust $H_{\infty}$ fault diagnosis for multi-model descriptor systems: A multi-objective approach, Proceedings of the 18th Mediterranean Conference on Control and Automation, Marrakech, Morocco, pp. 833-838.

Hamdi, H., Rodrigues, M., Mechmeche, C. and Braiek, N. B. (2012a). Robust fault detection and estimation for descriptor systems based on multi-models concept, International Journal of Control, Automation and Systems 10(6): 1260-1266.

Hamdi, H., Rodrigues, M., Mechmeche, C., Theilliol, D. and Braiek, N.B. (2012b). Fault detection and isolation in linear parameter-varying descriptor systems via proportional integral observer, International Journal of Adaptive Control and Signal Processing 26(3): 224-240.

Henry, D., Falcoz, A. and Zolghadri, A. (2009). Structured $H_{\infty} / H_{-}$LPV filters for fault diagnosis: Some new results, Proceedings of the 7th IFAC Symposium on Fault Detection, Supervision and Safety of Technical Processes, Barcelona, Spain, pp. 420-425.

Hou, M. and Patton, R.J. (1996). An LMI approach to $H_{-} / H_{\infty}$ fault detection observers, Proceedings of the UK Automatic Control Council International Conference on Control, Exeter, UK, pp. 305-310.

Ichalal, D., Marx, B., Ragot, J. and Maquin, D. (2009). Fault diagnosis in Takagi-Sugeno nonlinear systems, Proceedings of the 7th IFAC Symposium on Fault Detection, Supervision and Safety of Technical Processes, Barcelona, Spain, pp. 504-509.

Karimi, H.R. and Chadli, M. (2012). Robust observer design for Takagi-Sugeno fuzzy systems with mixed neutral and discrete delays and unknown inputs, Mathematical Problems in Engineering 2012: 1-13.

Koenig, D. (2005). Unknown input proportional multiple-integral observer design for linear descriptor systems: Application to state and fault estimation, IEEE Transactions on Automatic Control 50(2): 212-217. 
Koenig, D., Bedjaoui, N. and Litrico, X. (2005). Unknown input observers design for time-delay systems-application to an open-channel, Proceedings of the 44th IEEE Conference on Decision and Control, Seville, Spain, pp. 5794-5799.

Koumboulis, F.N., Fragkoulis, D.G. and Arsenos, P.I. (2011). Towards fault detection in singular Leontief production models, Proceedings of the 16th Conference on Emerging Technologies and Factory Automation, Toulouse, France, pp. 1-4.

Li, F. and Zhang, X. (2012). A delay-dependent bounded real lemma for singular LPV systems with time-variant delay, International Journal of Robust and Nonlinear Control 22(5): 559-574.

Liu, H., Shi, P., Karimi, H.R. and Chadli, M. (2016). Finite-time stability and stabilisation for a class of nonlinear systems with time-varying delay, International Journal of Systems Science 47(6): 1433-1444.

Lofberg, J. (2004). Yalmip: A toolbox for modeling and optimization in Matlab, Proceedings of the 2004 IEEE International Symposium on Computer Aided Control Systems Design, Taipei, Taiwan, pp. 284-289.

Lopez-Estrada, F.R., Ponsart, J.C., Theilliol, D. and Astorga-Zaragoza, C.M. (2014). Robust sensor FDI observer for polytopic descriptor LPV systems with unmeasurable scheduling varying parameter, American Control Conference (ACC), Portland, OR, USA, pp. 4452-4457.

Mazars, E., Jaimoukha, I.M. and Li, Z. (2008). Computation of a reference model for robust fault detection and isolation residual generation, Journal of Control Science and Engineering 2008: 1-6.

Meskin, N. and Khorasani, K. (2009). Robust fault detection and isolation of time-delay systems using a geometric approach, Automatica 45(6): 1567-1573.

Montes de Oca, S., Puig, V. and Blesa, J. (2012a). Robust fault detection based on adaptive threshold generation using interval LPV observers, International Journal of Adaptive Control and Signal Processing 26(3): 258-283.

Montes de Oca, S., Puig, V., Witczak, M. and Dziekan, Ł. (2012b). Fault-tolerant control strategy for actuator faults using LPV techniques: Application to a two degree of freedom helicopter, International Journal of Applied Mathematics and Computer Science 22(1): 161-171, DOI: 10.2478/v10006-012-0012-y.

Ocampo Martínez, C.A., Puig Cayuela, V., Cembrano Gennari, G. and Quevedo Casín, J.J. (2013). Application of predictive control strategies to the management of complex networks in the urban water cycle, IEEE Control Systems Magazine 33(1): 15-41.

Puig, V. and Blesa, J. (2013). Limnimeter and rain gauge FDI in sewer networks using an interval parity equations based detection approach and an enhanced isolation scheme, Control Engineering Practice 21(2): 146-170.
Puig, V., Cembrano Gennari, G., Romera, J., Quevedo Casín, J.J., Aznar, B., Ramón, G. and Cabot, J. (2009). Predictive optimal control of sewer networks using CORAL tool: Application to Riera Blanca catchment in Barcelona, Water Science and Technology 60(4): 869-878.

Saadni, M.S., Chaabane, M. and Mchdi, D. (2005). Stability and stabilizability of a class of uncertain dynamical systems with delays, International Journal of Applied Mathematics and Computer Science 15(3): 321-329.

Sadati, M., Hosseinzadeh, M. and Shafiee, M. (2013). Modelling and analysis of quadruped walking robot using singular system theory, Proceedings of the 21st Iranian Conference on Electrical Engineering, Mashhad, Iran, pp. 1-6.

Samiei, E. and Shafiee, M. (2010). Descriptor modeling and response analysis of two rigid-flexible cooperative arms, Proceedings of the 11th International Conference on Control, Automation, Robotics and Vision, Singapore, pp. 149-156.

Shamma, J.S. (1988). Analysis and Design of Gain Scheduled Control Systems, Ph.D. thesis, Massachusetts Institute of Technology, Cambridge, MA.

Share Pasand, M.M., Aliyari, M. and Taghirad, H.D. (2010) Unknown input proportional integral observer for singular systems: Application to fault detection, 18th Iranian Conference on Electrical Engineering, ICEE, Isfahan, Iran, pp. 662-667.

Sturm, J.F. (1999). Using Sedumi 1.02, a Matlab toolbox for optimization over symmetric cones, Optimization Methods and Software 11(1-4): 625-653.

Wang, J.L., Yang, G.H. and Liu, J. (2007). An LMI approach to $H_{-}$index and mixed $H_{-} / H_{\infty}$ fault detection observer design, Automatica 43(9): 1656-1665.

Wu, M., He, Y. and She, J.H. (2010). Stability Analysis and Robust Control of Time-delay Systems, Springer, Berlin.

Yao, X., Wu, L. and Zheng, W.X. (2011). Fault detection filter design for Markovian jump singular systems with intermittent measurements, IEEE Transactions on Signal Processing 59(7): 3099-3109.

Yeu, T.K., Kim, H.S. and Kawaji, S. (2005). Fault detection, isolation and reconstruction for descriptor systems, Asian Journal of Control 7(4): 356-367.

Youssef, T., Chadli, M., Karimi, H.R. and Zelmat, M. (2014). Design of unknown inputs proportional integral observers for TS fuzzy models, Neurocomputing 123: 156-165.

Zhai, D., Zhang, Q.L. and Li, J.H. (2014). Fault detection for singular multiple time-delay systems with application to electrical circuit, Journal of the Franklin Institute 351(12): 5411-5436.

Zhang, X. and Zhu, H. (2012). Robust stability and stabilization criteria for discrete singular time delay LPV systems, Asian Journal of Control 14(4): 1084-1094. 


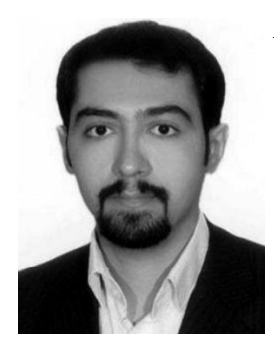

Amir Hossein Hassanabadi was born in 1985. He received his B.Sc. degrees in control systems engineering and electronics in 2007 and 2009, respectively, both from the Amirkabir University of Technology (Tehran Polytechnic), Tehran, Iran. He received his M.Sc. degree in control engineering in 2010 from the same university, where he is currently pursuing his Ph.D. degree in control systems engineering. His present research interests include singular systems, robotics, timedelay systems, and fault diagnosis.

Masoud Shafiee was born in 1957. He received a B.Sc. degree in mathematics in 1975, an M.Sc. degree in mathematics and in systems engineering from Wright State University, Dayton, OH, in 1980 and 1982, respectively, and M.Sc. and Ph.D. degrees in electrical engineering from Louisiana State University, Baton Rouge, LA, in 1984 and 1987, respectively. M. Shafiee is currently a full professor in the Department of Electrical Engineering at the Amirkabir University of Technology, Tehran, Iran. Also, he joined the SIC Research Institute in 2014. He is the author of over 230 research papers and 13 books, and a translator of 11 books. His research interests include multi-dimensional (M-D) systems, singular systems, information and communications, robotics, and network stability.

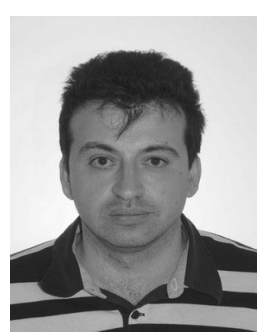

Vicenc Puig was born in Girona, Spain, in 1969, received the $\mathrm{Ph} . \mathrm{D}$. degree in control engineering in 1999 and the telecommunications engineering degree in 1993, both from Universitat Politècnica de Catalunya (UPC), Barcelona, Spain. He is currently a professor of automatic control and the leader of the Advanced Control Systems (SAC) Research Group of the Research Center for Supervision, Safety and Automatic Control (CS2AC) at UPC. His main research interests include fault detection, isolation and fault-tolerant control of dynamic systems. He has been involved in several European projects and networks, and has published many papers in international conference proceedings and scientific journals.

Received: 2 November 2014

Revised: 12 April 2015

Re-revised: 22 July 2015 